\title{
A REMARK ON THE ORTHOGONALITY RELATIONS IN THE REPRESENTATION THEORY OF FINITE GROUPS
}

\author{
HIROSI NAGAO
}

Let $G$ be a finite group of order $g$, and

$$
t \rightarrow\left(a_{i j}^{(\mu)}(t)\right) \quad(\mu=1,2, \ldots, k)
$$

be an absolutely irreducible representation of degree $f_{\mu}$ over a field of characteristic zero. As is well known, by using Schur's lemma (1), we can prove the following orthogonality relations for the coefficients $a_{i j}{ }^{(\mu)}(t)$ :

$$
\sum_{l \in G} a_{i j}^{(\mu)}(t) a_{k l}^{(\nu)}\left(t^{-1}\right)=\delta_{\mu \nu} \delta_{i l} \delta_{j k} \frac{g}{f_{\mu}} .
$$

It is easy to conclude from (1) the following orthogonality relations for characters:

$$
\begin{aligned}
& \sum_{t \in G} \chi^{(\mu)}(t) \chi^{(\nu)}\left(t^{-1}\right)=\delta_{\mu \nu} g \\
& \sum_{\mu=1}^{k} \chi^{(\mu)}(t) \chi^{(\mu)}\left(s^{-1}\right)=\delta_{t, s} n(t)
\end{aligned}
$$

where

$$
\chi^{(\mu)}(t)=\sum_{i} a_{i i}^{(\mu)}(t),
$$

and $\bar{\delta}_{t, s}$ is 1 or 0 according as $t$ and $s$ are conjugate in $G$ or not, and $n(t)$ is the order of the normalizer of $t$.

In this short note, we remark that we can conclude (1) from (3) or from a special case of $(3)$ :

$$
\sum_{\mu=1}^{k} f_{\mu} \chi^{(\mu)}(t)=\delta_{1, t} g
$$

Let us now asume $\left(3^{\prime}\right)$. Setting $t=1$ in $\left(3^{\prime}\right)$ we have

$$
g=\sum_{\mu} f_{\mu}^{2}
$$

Therefore the number of $(\mu ; i, j)$ such that $1 \leqslant \mu \leqslant k$ and $1 \leqslant i, j \leqslant f_{\mu}$ is $g$. Let $A$ be the matrix of degree $g$ with the row index $t$, column index $(\mu ; i, j)$ and $(t,(\mu ; i, j))$-element $a_{i, j}{ }^{(\mu)}(t)$.

Let $B$ be the matrix with row index $(\mu ; i, j)$, column index $t$ and $((\mu ; i, j), t)$ element

Received April 23, 1958.

$$
\frac{f_{\underline{\mu}}}{g} \cdot a_{j i}^{(\mu)}\left(t^{-1}\right)
$$


The $(t, s)$-element in $A B$ is

$$
\begin{aligned}
& \sum_{\mu, i, j} a_{i, j}^{(\mu)}(t) \cdot \frac{f_{\mu}}{g} a_{j i}^{(\mu)}\left(s^{-1}\right) \\
& \quad=\sum_{\mu, i} \frac{f_{\mu}}{g} a_{i i}^{(\mu)}\left(t s^{-1}\right)=\frac{1}{g} \cdot \sum_{\mu} f_{\mu} \cdot \chi^{(\mu)}\left(t s^{-1}\right)=\delta_{\iota, s} .
\end{aligned}
$$

This shows that $A B=E$, and hence $B A=E$.

Since the $((\mu ; i, j),(\nu ; k, l))$-element of $B A$ is

$$
\frac{f_{\mu}}{g} \sum_{t \in G} a_{i j}^{(\mu)}(t) a_{k l}^{(\nu)}\left(t^{-1}\right)
$$

we have (1).

\section{REFERENCE}

1. I. Schur, Neue Begründung der Theorie der Gruppencharactere, Sitzungsber. Preus. Akad. (1. Wiss. (1905), 406-32.

Osaka City University

The University of Toronto 